\title{
Effect of Nanoparticle Morphologies on Signal Strength in Photoacoustic Sensing
}

\author{
Craig S. Murdoch*, Jonas Kusch*, Gordon M. H. Flockhart*, Duncan Graham**, Karen Faulds**, Deepak \\ Uttamchandani* \\ *Centre for Microsystems and Photonics, \\ ***entre for Molecular Nanometrology, \\ University of Strathclyde, \\ Glasgow, UK.
}

\begin{abstract}
Spherical gold nanoparticles with a plasmonic extinction peak at $532 \mathrm{~nm}$ and two sizes of star shaped gold nanoparticles with plasmonic extinction peaks at $532 \mathrm{~nm}$ and 600 $\mathrm{nm}$ were synthesised and introduced into tissue phantoms as exogenous absorbers. The photoacoustic signals generated from the three different nanoparticle morphologies embedded in tissue the phantoms is compared. The effect of nanoparticle concentration on the generated photoacoustic signal strength was also investigated for the spherical nanoparticles. At an excitation laser wavelength of $532 \mathrm{~nm}$, the spherical gold nanoparticles were shown to produce the greatest photoacoustic response.
\end{abstract}

Keywords-photoacoustic; gold nano-stars; gold nano-spheres; nanoparticles;

\section{INTRODUCTION}

Photoacoustic sensing and imaging are increasingly taking advantage of plasmonic nanoparticles as exogenous contrast agents. An important factor fuelling this trend is the relative ease with which plasmonic resonances of metallic nanoparticles, e.g. gold nanoparticles, can be engineered to align with commonly used visible and near infra-red laser wavelengths, thereby increasing the overall optical-to-acoustic conversion efficiency using affordable laser systems [1]. Building upon the optimization of this photoacoustic efficiency, the next evolutionary step is to use functionalized metallic nanoparticles so that very specific nanobiological and molecular interactions occurring on the surface of the nanoparticle can be photoacoustically probed [2].

Compared to alternative all-optical based probing techniques used for sensing changes in biological tissue, such as fluorescence or Raman microscopy which require bi-directional propagation of light, the photoacoustic based technique has the advantage of requiring uni-directional light propagation, and therefore the adverse impact of light scattering in tissue on the detected signal is significantly reduced [3]. Consequently, photoacoustic sensing, mediated by functionalized nanoparticles, has the potential to probe nanobiological and molecular interactions from, generally greater depth - up to a few centimeters in biological tissue - compared to the microscopies mentioned above [4], and this has recently generated considerable interest in this technique. This paper reports work on in-house synthesis of (non-functionalized) gold nanoparticles of different geometrical shapes and optical extinction profiles, and subsequently compares their photoacoustic signal response at a fixed wavelength using a laser-excited and piezo-transducer based photoacoustic sensing system.

\section{SYNTHESIS AND OPTICAL}

\section{CHARACTERISATION OF NANOPARTICLES}

\section{A. Synthesis Steps}

Colloidal gold nanoparticles were prepared in both spherical and star shapes. Star shaped gold nanoparticles have a lower molar extinction coefficient than spheres but their absorption peak can be tuned to longer wavelengths thereby enabling photoacoustic sensing systems to employ lower cost infra-red laser diode sources [5]. The star shaped nanoparticles were synthesised in two different sizes. Gold stars were synthesised using a facile, "one-pot" process combining a growth solution, gold nanoparticle seeds and a reducing agent. The growth solution is a combination of sodium tetrachloroaurate and silver nitrate. A solution of ascorbic acid was used as the reducing agent and $12 \mathrm{~nm}$ gold nanoparticles where used as star seeds. The spikes of the star grow on top of the seed and the final star size, and peak extinction wavelength, is dictated by the ratio of growth solution to seed. A higher growth solution to seed ratio will produce longer spikes and a longer peak absorption wavelength. Spherical gold nanoparticles were prepared using a simple sodium citrate reduced synthesis method [6].

\section{B. Optical Characterisation}

The optical properties of each shape and size of nanoparticle was investigated using ultraviolet-visible spectroscopy. Fig. 1 shows the extinction spectra of the two different sizes of stars. One size of star nanoparticle has a peak extinction wavelength at $532 \mathrm{~nm}$ and a FWHM of $100 \mathrm{~nm}$. The second star size has a peak extinction at $600 \mathrm{~nm}$ and a FWHM of $350 \mathrm{~nm}$. This indicates a star diameter of approximately 15 $\mathrm{nm}$ with spike lengths of $\sim 3 \mathrm{~nm}$ and $\sim 6 \mathrm{~nm}$ respectively [5]. The dashed line plots in Fig. 1 show the extinction spectra of nanoparticle doped gelatin. The decreased extinction is due to a decrease in concentration because of the addition of gelatin. This indicates that the addition of nanoparticles to gelatin does not alter their optical properties. Fig. 2. shows the extinction spectra of the spherical gold nanoparticles. These nanoparticles

This work was supported by the Engineering and Physical Sciences Research Council and Medical Research Council [grant number EP/L016559/1] 
have a peak extinction wavelength at $532 \mathrm{~nm}$ and a FWHM of $150 \mathrm{~nm}$. The diameter of the spherical nanoparticles has been confirmed to be around $45 \mathrm{~nm}$ by SEM which agrees with the peak absorption [1], [7].

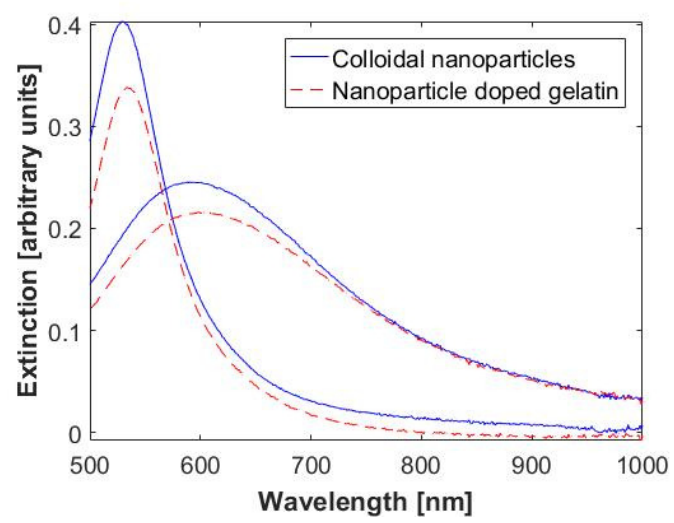

Fig. 1. Extinction spectra of two different sizes of star shaped gold nanoparticles in colloid (solid line) and in gelatin (dashed line).

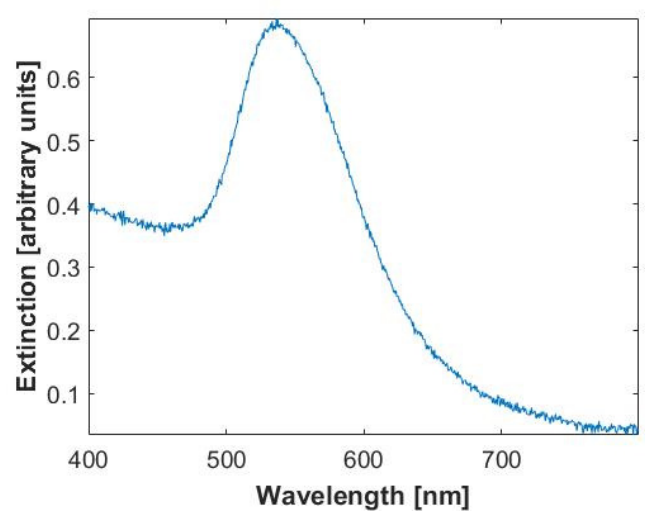

Fig. 2. Extinction spectrum of spherical gold nanoparticles.

\section{EXPERIMENTAL APPROACH}

\section{A. Fabrication of Phantoms}

Tissue phantoms made of gelatin were used to evaluate and compare the photoacoustic response of each type of nanoparticle. The phantoms are composed of $10 \%$ porcine gelatin so that they mimic the mechanical properties of real tissue. They are doped with nanoparticles to enhance their optical absorption properties and in turn enhancing their opticalto-acoustic conversion. Phantoms were prepared by dissolving porcine gelatin powder into the nanoparticle colloid which was heated using a water bath. This mixture was then poured into a mould and refrigerated to set. Visual inspection of the cured phantoms indicates uniform distribution of nanoparticles. Cube shaped phantoms of dimension $1 \mathrm{~cm}^{3}$ and $4 \mathrm{~cm}^{3}$ have been made using custom moulds. Three $4 \mathrm{~cm}^{3}$ phantoms were made and each was doped with $9 \mu \mathrm{gml}^{-1}$ of the spherical nanoparticles or one of the two sizes of star shaped nanoparticles. As shown in Fig. 3, six $1 \mathrm{~cm}^{3}$ phantoms were also produced and doped with different concentrations of spherical nanoparticles from 30 $\mu \mathrm{gml}^{-1}$ to $5 \mu \mathrm{gml}^{-1}$. Each phantom was removed from refrigeration and allowed to heat up to room temperature before any measurements were taken.

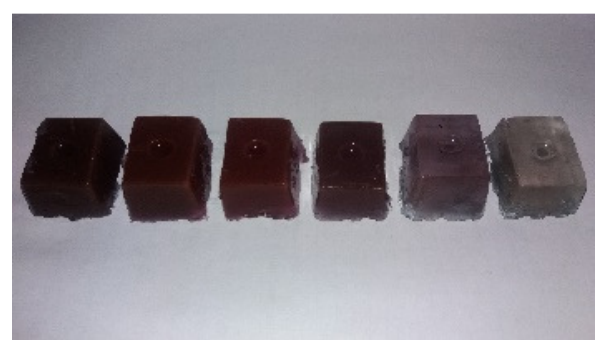

Fig. $3.1 \mathrm{~cm}^{3}$ tissue phantoms with concentrations $30 \mu \mathrm{gml}^{-1}, 25$ $\mu \mathrm{gml}^{-1}, 20 \mu \mathrm{gml}^{-1}, 15 \mu \mathrm{gml}^{-1}, 10 \mu \mathrm{gml}^{-1}$ and $5 \mu \mathrm{gml}^{-1}$, from left to right, of the spherical nanoparticles.

\section{B. Optical Characterisation}

The photoacoustic sensing arrangement consists of three parts; a pulsed excitation laser for generation of photoacoustic ultrasound, an ultrasonic transducer for detection of the ultrasonic signals and an oscilloscope which doubled as a fast analogue-to-digital converter for acquisition and digitization of the ultrasonic signals. A frequency doubled, pulsed Nd:YAG laser with a wavelength of $532 \mathrm{~nm}$ producing pulses of $10 \mathrm{~ns}$ duration was used as the excitation laser. A piezoelectric transducer with a central frequency of $10 \mathrm{MHz}$ and a bandwidth of $10 \mathrm{MHz}$ has been used to detect ultrasound from inside the tissue phantoms. An Agilent Infiniium oscilloscope has been used for acquisition of photoacoustic signals using a high speed photodiode to produce a trigger signal from the laser pulse. To compare the photoacoustic signal strength of the three different nanoparticle morphologies, the laser pulse energy used was 23 $\mathrm{mJ}$ with a shot to shot standard deviation of $3.5 \mathrm{~mJ}$. For measurements of the photoacoustic signal strength using different spherical nanoparticle concentrations the laser pulse energy used was lower at $14.2 \mathrm{~mJ}$ with a standard deviation of $0.186 \mathrm{~mJ}$.

\section{RESULTS AND DISCUSSION}

A $70 \mathrm{~mm}$ focal length lens was used to focus the $532 \mathrm{~nm}$ Nd:YAG laser inside the gelatin based phantom generating a photoacoustic signal. The $10 \mathrm{MHz}$ transducer was acoustically coupled to a perpendicular face of the phantom with ultrasound gel. For each phantom, 16 ultrasound waveforms, Fig. 4, were acquired and their average calculated. The digitised signals are then transferred to a computer for processing. The photoacoustic response of each type of nanoparticle has been quantified by taking the absolute value of the Hilbert transform of each ultrasound waveform to calculate a waveform envelope. Each point on this envelope was then summed to give a single value which represents the photoacoustic signal strength. For identical illumination conditions, Table 1 shows that the spherical gold nanoparticles produced an average photoacoustic signal strength of 6.4 a.u. The star shaped gold nanoparticles with extinction peaks at $532 \mathrm{~nm}$ and $600 \mathrm{~nm}$ produced photoacoustic signal strengths of 4.7 a.u. and 3.9 a.u. respectively. Fig. 5 shows the change in photoacoustic signal strength as the concentration of 
the gold spherical nanoparticles is changed from 0 to $30 \mu \mathrm{gml}^{-1}$. As can be seen the signal strength sharply increases from 5 a.u. to 10 a.u. from the un-doped reference gelatin phantom $(0$ $\mu \mathrm{gml}^{-1}$ ) to the doped sample with $5 \mu \mathrm{gml}^{-1}$. From $5 \mu \mathrm{gml}^{-1}$ to 30 $\mu \mathrm{gml}^{-1}$ the photoacoustic signal strength increases more slowly from 10 a.u. to 13 a.u.

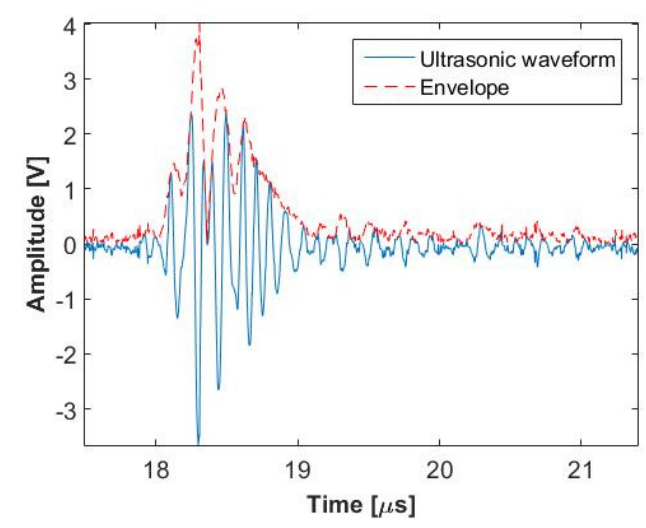

Fig. 4. Example of an ultrasound waveform (solid line) acquired using the photoacouistic sensing system and the waveform envelope (dashed line) calculated by taking the absolute value of the Hilbert transform.

Table 1. Signal strength of the photoacoustic response of spherical gold nanoparticles with a peak extinction at $532 \mathrm{~nm}$ and two sizes of star shaped gold nanoparticles with peak extinctions at $532 \mathrm{~nm}$ and $600 \mathrm{~nm}$.

\begin{tabular}{|c|c|c|c|}
\hline Shape & Sphere & Star & Star \\
\hline Peak Extinction [nm] & $\mathbf{5 3 2}$ & $\mathbf{5 3 2}$ & $\mathbf{6 0 0}$ \\
\hline Signal Strength [arb. units] & $\mathbf{6 . 4}$ & $\mathbf{4 . 7}$ & $\mathbf{3 . 9}$ \\
\hline Standard Deviation & $\mathbf{0 . 7}$ & $\mathbf{0 . 4}$ & $\mathbf{0 . 3}$ \\
\hline
\end{tabular}

The star shaped gold nanoparticles that have an extinction peak at $532 \mathrm{~nm}$ are resonant with the emission wavelength of the excitation laser. This is the reason they generated a greater photoacoustic signal strength compared to the star shaped nanoparticles with an extinction peak at $600 \mathrm{~nm}$. The $532 \mathrm{~nm}$ spherical nanoparticles produced the greatest photoacoustic signal strength as they have a molar extinction coefficient two orders of magnitude greater than the stars and so absorb light more effectively. Although the spherical nanoparticles generated a higher photoacoustic response at a laser wavelength of $532 \mathrm{~nm}$ this may not translate to higher wavelengths. Unlike star shaped nanoparticles, spherical nanoparticles are limited to a maximum extinction peak of $600 \mathrm{~nm}$.

The first data point in Fig. 5 corresponds to a control phantom where the concentration of spherical gold nanoparticles is zero. It can be seen that the inherent absorption properties of the gelatin produced a small photoacoustic signal strength of 5 a.u. To establish a clear trend more measurements need to be obtained between the concentrations of 0 and $5 \mu \mathrm{gml}^{-1}$. From 5

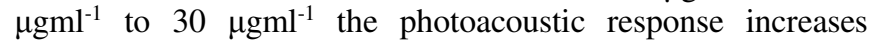
linearly.

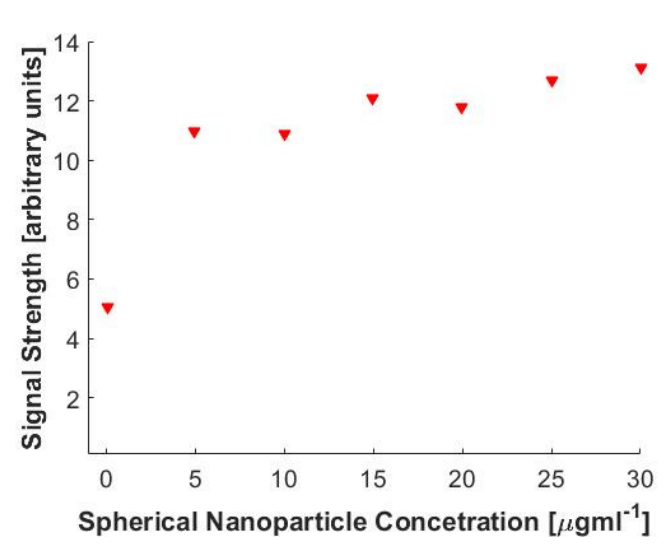

Fig. 5. Signal strength of photoacoustic response of spherical gold nanoparticles for concentrations from 0 to $30 \mu \mathrm{gml}^{-1}$.

\section{CONCLUSION}

An in-house photoacoustic sensing setup has been used to compare the photoacoustic response of three different nanoparticle morphologies. One size of spherical gold nanoparticles and two sizes of star shaped gold nanoparticles have been synthesised and characterised when embedded in a tissue phantom. It was found that at a laser wavelength of $532 \mathrm{~nm}$ the spherical nanoparticles generated the strongest photoacoustic signal strength. It has also been shown that the photoacoustic signal approximately doubles when comparing an un-doped phantom to a $5 \mathrm{\mu gml}^{-1}$ doped phantom. As the

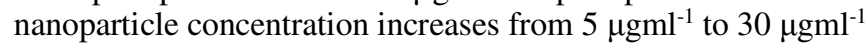
the photoacoustic signal strength generated continues to increase linearly but at a slower rate.

\section{ACKNOWLEDGEMENT}

This work has been a collaboration between OPTIMA, the Centre for Microsystems and Photonics and the Centre for Molecular Nanometrology, University of Strathclyde, UK.

\section{REFERENCES}

[1] A. Feis, C. Gellini, P. R. Salvi, and M. Becucci, "Photoacoustic excitation profiles of gold nanoparticles," Photoacoustics, vol. 2, no. 1, pp. 47-53, 2014.

[2] J. Weber, P. C. Beard, and S. E. Bohndiek, "Contrast agents for molecular photoacoustic imaging," Nat. Publ. Gr., vol. 13, no. 8, pp. 639-650, 2016.

[3] S. L. Chen, L. J. Guo, and X. Wang, "All-optical photoacoustic microscopy," Photoacoustics, vol. 3, no. 4, pp. 143-150, 2015.

[4] L. V Wang et al., "Photoacoustic tomography: in vivo imaging from organelles to organs.," Science, vol. 335, no. 6075, pp. 1458-62, 2012.

[5] H. de Puig, J. O. Tam, C.-W. Yen, L. Gehrke, and K. HamadSchifferli, "Extinction Coefficient of Gold Nanostars," J. Phys. Chem. C, vol. 119, no. 30, pp. 17408-17415, 2015.

[6] P. C. Stevenson, "A STUDY OF THE NUCLEATION AND GROWTH PROCESSES I N THE SYNTHESIS OF,” vol. 55, no. c, 1951.

[7] P. K. Jain, K. S. Lee, I. H. El-Sayed, and M. A. El-Sayed, "Calculated absorption and scattering properties of gold nanoparticles of different size, shape, and composition: Applications in biological imaging and biomedicine," J. Phys. Chem. B, vol. 110, pp. 7238-7248, 2006. 